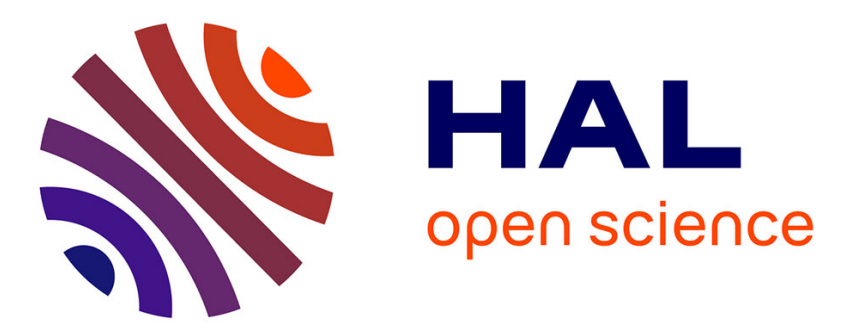

\title{
Pérennité et invariants dans la construction des savoirs géographiques \\ Yann Calberac
}

\section{To cite this version:}

Yann Calberac. Pérennité et invariants dans la construction des savoirs géographiques: Construction, transmission et adaptation d'un habitus du terrain dans la géographie française. Martine Tabeaud. Le changement en environnement. Les faits, les représentations, les enjeux, Publications de la Sorbonne, pp.93-107, 2009, 10.4000/books.psorbonne.37521 . halshs-00369784

\section{HAL Id: halshs-00369784 https://shs.hal.science/halshs-00369784}

Submitted on 21 Mar 2009

HAL is a multi-disciplinary open access archive for the deposit and dissemination of scientific research documents, whether they are published or not. The documents may come from teaching and research institutions in France or abroad, or from public or private research centers.
L'archive ouverte pluridisciplinaire HAL, est destinée au dépôt et à la diffusion de documents scientifiques de niveau recherche, publiés ou non, émanant des établissements d'enseignement et de recherche français ou étrangers, des laboratoires publics ou privés. 


\title{
Pérennité et invariants dans la construction des savoirs géographiques Construction, transmission et adaptation d'un habitus du terrain dans la géographie française
}

\author{
Yann Calbérac \\ Université de Lyon (Université Lumière Lyon 2) \\ CNRS UMR 5600 « Environnement, ville, société »
}

L'histoire de la géographie française est caractérisée par deux mouvements opposés. D'une part, l'évolution de la discipline obéit à un principe de continuité : les positions acquises à l'Université, à l'école ou dans la société depuis Vidal de La Blache et ses disciples à l'aube du $\mathrm{XX}^{\mathrm{e}}$ siècle ne sont pas contestées, plus d'un siècle après. Mais d'autre part, la géographie n'échappe pas à des remises en cause régulières de ses objets et de ses méthodes: dans les années 1960 et 1970, des débats internes à la discipline remettent en cause l'héritage classique et la pertinence de ses objets. Cette crise de la géographie a entrainé le renouvellement en profondeur des approches, l'apparition de nouveaux objets, la diffusion de méthodes innovantes et la reformulation des paradigmes fondateurs de la discipline. Les recherches récentes invitent à chercher les facteurs de rupture ou d'unité au-delà de ce clivage, dans les incessantes interrogations des géographes sur leur discipline: selon Marie-Claire Robic, la géographie française, depuis son institutionnalisation par Vidal de La Blache, « se pose, de manière plus ou moins récurrente, les questions de son identité intellectuelle et de sa pertinence sociale ». (Robic (dir.), 2006: 9). Ce constat rétrospectif postule l'existence d'un «grand $\mathrm{XX}^{\mathrm{e}}$ siècle de géographie » (Ibid.) qui court des années 1890 au début des années 2000 et apporte un éclairage nouveau sur l'histoire de la discipline et l'évolution de ses méthodes et de ses objets. Une telle formule suggère en effet que la continuité l'emporte sur les ruptures et que les mêmes interrogations réflexives continuent d'être des moteurs du renouvellement disciplinaire.

C'est sous l'angle du terrain, c'est-à-dire l'un des gestes constitutifs du métier de géographe, que nous interrogerons les facteurs de changement et de continuité qu'a connus la géographie française depuis un siècle. En effet, la géographie qu'impose Vidal de La Blache propose un renouveau des méthodes lié à de nouveaux objets et à de nouvelles échelles d'analyse : la géographie devient régionale et le géographe sort de son cabinet pour entrer au contact de la réalité empirique étudiée (Lefort \& Pelletier, 2006). La pratique du terrain est donc consubstantielle aux efforts effectués pour moderniser la géographie, la rendre opératoire, fonder ses objets et asseoir sa légitimité institutionnelle et son autorité scientifique. En dépit des débats qui aboutissent aujourd'hui à une diversité des voix, le terrain et sa pratique n'ont pas été durablement remis en cause par l'ensemble de la communauté scientifique, si bien que le terrain peut apparaître comme un facteur de continuité dans l'histoire de la géographie française. C'est cette surprenante pérennité dans une discipline qui a pourtant connu des bouleversements en profondeur qu'il faut interroger : l'ordinateur, les statistiques et les SIG ne semblent pas avoir eu raison du terrain.

L'hypothèse débattue ici est que le terrain et sa pratique fonctionnent, tout au long du siècle, comme un habitus d'autant plus durable qu'il a été profondément installé dans les usages et enraciné dans les représentations des géographes. Envisager le terrain comme un habitus nous oblige donc à étudier son contenu et son fonctionnement, ainsi que les lieux et les temps de sa production, de sa transmission et de son acquisition, et à confronter cet ensemble cohérent aux évolutions de la discipline dans le long terme. Pour remplir ce programme, il faut donc s'intéresser à la formation au terrain dispensée à l'Université : c'est en effet dans leur dimension pédagogique que les pratiques de terrain sont mises en scène, s'explicitent et se transmettent. Pour cela, nous croiserons deux corpus qui nous donnent à voir la formation des géographes à 
l'Université, la transmission des gestes du métier et la construction de l'habitus et des représentations du terrain.

Le premier corpus est constitué par l'ensemble des comptes rendus des excursions interuniversitaires publiés chaque année dans les Annales de géographie de 1906 à 1973. Ces excursions, entamées dès 1905 en Bretagne, sont nées de l'initiative d'Emmanuel de Martonne, Albert Demangeon et Lucien Gallois ; elles ont alors pour but de promouvoir la pratique du terrain dans les pratiques pédagogiques et de récompenser les étudiants les plus méritants de chaque université (Wolff, 2001). En dépit des biais qu'entraîne la mise en texte de excursions, ces comptes rendus permettent, dans une certaine mesure, de reconstituer le déroulement des excursions et, par là, de comprendre les modalités de la formation dispensée sur le terrain (Calbérac, 2005). Le second corpus rassemble quant à lui les documentaires géographiques produits à l'Ecole normale supérieure de Saint-Cloud à destination des étudiants. Le Centre audio-visuel, fondé en 1953 par Robert Lefranc pour réfléchir aux usages didactique du cinéma scolaire et produire des films pour les classes primaires et secondaires, se réoriente en effet en 1969 vers la production des seuls films destinés à l'enseignement supérieur dans le contexte de sa massification et de la mise en place de l'enseignement à distance (Dubost, 2004). Des universitaires renommés (parmi lesquels Philippe Pinchemel, Fernand Verger, Etienne Juillard, Jean Tricart...) sont alors régulièrement appelés à produire jusqu'en 1995 une vingtaine de films à l'intérêt pédagogique et scientifique incontestable et qui constituent des jalons dans l'histoire de la géographie, au moment même où cette dernière affronte sa crise.

Ces corpus présentent de nombreux intérêts et leur croisement est fécond. D'une part, la durée couverte (1905-1995) est exceptionnelle: on approche presque le "grand $\mathrm{XX}^{\mathrm{e}}$ siècle de géographie française »; l'habituelle coupure des années 1960 et 1970 est bien représentée et se traduit ici par l'utilisation de nouveaux supports pédagogiques et de nouvelles méthodes de transmission des savoirs. D'autre part, même si les objectifs de ces deux types de documents ne sont pas les mêmes (témoignages d'un événement pour les comptes rendus d'excursions, matériel pédagogique pour les films), une certaine homogénéité apparaît dans leur contenu, dans la mesure où les uns et les autres présentent des figures de géographes et des postures scientifiques mobilisées sur le terrain à des fins de formation. Ils permettent donc d'articuler les pratiques pédagogiques des géographes présentés dans les corpus à leurs pratiques scientifiques. Enfin, cet ensemble très riche permet d'avoir un bon panorama des acteurs - enseignants et étudiants - de la géographie française et des lieux de production des savoirs sur un siècle.

\section{Le terrain : un ensemble de geste structuré et structurant du métier de géographe}

En quoi le terrain constitue-t-il un habitus au sens que lui donne Pierre Bourdieu, c'est-à-dire un «système de dispositions durables et transposables, structures structurées prédisposées à fonctionner comme structures structurantes, c'est-à-dire en tant que principes générateurs et organisateurs de pratiques et de représentations (...)» (Bourdieu, 1980:88) ? Pour élucider son fonctionnement, nous devons donc nous intéresser aux modalités de construction, de diffusion, d'acquisition et d'assimilation des pratiques de terrain, constitutives à nos yeux d'un habitus partagé par l'ensemble de la communauté. Il faut ainsi étudier cet ensemble de pratiques - dont l'explicitation est indispensable - en tant qu'il structure la communauté des géographes qui s'y reconnaissent. Pourquoi l'accession à la communauté impose-t-elle nécessairement l'assimilation des règles et des pratiques du terrain ? A l'inverse, en quoi notre approche et notre conception du terrain peuvent-elles être enrichies par un recours au concept d'habitus?

\section{Le terrain comme lieu de déploiement de pratiques constitutives d'un habitus}

L'ambiguïté du terme terrain vient de ce qu'il désigne à la fois un fragment d'espace objectivé par un géographe engagé dans une démarche heuristique, ainsi que l'ensemble des pratiques 
empiriques qu'il y déploie à cette occasion (Volvey, 2003). Ces deux instances sont indissociables et sont conjointement constitutives l'une de l'autre : sans espace à étudier, aucun geste n'est déployé ; et sans geste spécifique, aucun moyen d'étudier un espace. Le regard objectivant porté par le géographe sur l'espace entraîne aussitôt l'adoption d'une posture adaptée et de pratiques appropriées. Ces deux corpus présentent ainsi un grand nombre de gestes communément déployés par les géographes sur leurs terrains et dont l'éventail recoupe le binôme mis en évidence par Anne Volvey (Volvey, 2003) : l'observation et l'enquête, c'est-àdire le recueil d'une information qui d'un côté se donne et que de l'autre il faut aller chercher. Cela implique différentes postures dans les protocoles d'observation.

Le mode d'observation privilégié est l'autopsie (c'est-à-dire la description et l'analyse d'un paysage en vue directe); elle est bien représentée dans les excursions et même dans certains films (Anatomie du paysage de Philippe Pinchemel, Stage d'étude sur le terrain en géographie de Jean Tricart). Elle correspond à une innovation méthodologique de la géographie vidalienne et justifie la nécessité même de faire du terrain (Robic, 1996). Elle requiert des points élevés (naturels ou bâtis) qui permettent au géographe de dominer (au sens propre comme au sens figuré) son terrain. Dans la tradition vidalienne, ce commentaire de paysage permet de dégager la physionomie de la région et de dégager les principaux traits de son organisation, tant physiques qu'humains.

L'autopsie surplombante est complétée par une observation au sol, au niveau des objets étudiés : le pied d'un talus (Stage d'étude sur le terrain en géographie de Jean Tricart), un marais littoral (Pixels et paysages de Fernand Verger), un lotissement (Pathologie du paysage de Philippe Pinchemel)... C'est près du sol en effet que l'on peut recueillir des informations, c'est-à-dire passer de l'observation à l'enquête. Le film Des géographes de Jean-Louis Tissier constitue une belle collection de ces pratiques destinées à recueillir des données parmi lesquelles on retrouve le travail de cartographie mené par Claude Collin-Delavaud sur les rives de l'Amazone ou les mesures fines sur les milieux naturels effectués par François Morand et son équipe dans la station expérimentale de Cessières dans l'Aisne.

Ces corpus recèlent aussi un protocole à mi-chemin entre l'observation et l'enquête : il s'agit de l'observation directe, méthode privilégiée du sociologue et de l'ethnologue, qui consiste pour le scientifique à s'immerger dans un groupe et à tirer de l'observation des interactions quotidiennes des éléments permettant de comprendre le fonctionnement du groupe dans son ensemble. L'observateur cherche de la signification dans ce que le groupe donne à voir de son quotidien. C'est cette méthode qu'adopte Bernard Kayser pour comprendre l'impact de la périurbanisation dans une exploitation agricole de la campagne toulousaine (Un tracteur de banlieue): si le film est scénarisé, les scènes montrées, tirées du quotidien de la famille observée, se font l'écho des préoccupations du moment, c'est-à-dire l'étalement urbain et son impact sur l'agriculture dans la périphérie des villes.

Enfin, l'observation est souvent complétée par des entretiens avec des personnes ressources. C'est une autre manière d'accéder à l'information, surtout quand l'information pertinente change de nature en même temps que la discipline évolue. Le film Qu'est-ce qu'une région? Un exemple: la région de Strasbourg a recours à cette forme de collecte de données particulièrement télégénique une fois posée l'aporie de la démarche classique fondée sur l'observation des paysages et la physionomie des contrées. Pour définir la région strasbourgeoise, Etienne Julliard ne s'intéresse plus guère aux paysages de l'Alsace mais interroge successivement le directeur d'une succursale bancaire, un journaliste aux Dernières nouvelles d'Alsace, le responsable d'une salle de spectacles et celui d'une coopérative d'achat. De ces entretiens il tire des cartes qui montrent les réseaux qui permettent à Strasbourg de polariser sa région. 
La mise en pratique de ces gestes tout au long de la période couverte souligne leur fonction unificatrice en terme de pratiques et de représentations qu'ils suscitent : ils sont assimilés et mis en œuvre par des géographes qui les jugent toujours scientifiquement féconds.

\section{Le terrain comme lieu de la reconnaissance institutionnelle}

Les approches récentes en sociologie des sciences (Latour, 1993 et 2005) placent au cœur des pratiques des chercheurs la question de la légitimité scientifique et de la reconnaissance institutionnelle : toute production de savoirs permet au chercheur qui les formule d'accroître sa légitimité (qui autorise ses jugements) et assoit sa réputation et la notoriété de ses travaux. Le travail du chercheur consiste donc à accroître son capital de légitimité qu'il peut ensuite valoriser dans le meilleur jugement porté par ses collègues ou dans toute autre opportunité de carrière. Le terrain est le principal élément dont le géographe tire sa légitimité (pour s'en convaincre, il suffit de rappeler le déterminant possessif que les géographes accolent souvent à leur terrain et qui traduit bien l'appropriation scientifique, parfois exclusive, qui unit le géographe à son terrain); il participe donc pleinement du processus de fonctionnement et d'institutionnalisation de la communauté scientifique : le terrain permet de témoigner et de fonder la légitimité du géographe. Cette dernière repose en effet d'abord sur la bonne connaissance par le géographe du terrain qu'il étudie, mais aussi, dans une certaine mesure, sur la mise en œuvre sur ce terrain des gestes appropriés, issus de l'attirail méthodologique et pratique commun aux géographes.

Les terrains parcourus dans ces corpus sont donc indissociables des géographes illustres qui les ont étudiés et fait connaître. En creux se dessine la figure du maître, reconnu par ses pairs et ses étudiants et dont la compétence et le prestige sont associés à un terrain sur lequel il manifeste l'étendue de ses talents. C'est une galerie des portraits de quelques-uns de ces grands maîtres, associés à leurs terrains et à leurs méthodes, que l'on parcourt au gré de ces corpus. Ainsi, les excursions interuniversitaires commencent-elles en 1905, en Bretagne, sur les terres d'Emmanuel de Martonne alors en poste à l'Institut de géographie de Rennes qu'il a fondé ; l'itinéraire qu'il a mis au point entraîne la caravane dans le relief appalachien du sud de Rennes dont il a proposé une explication. Ses qualités d'infatigable marcheur sont mises en avant et les participants ont parfois du mal à suivre. Raoul Blanchard organise quant à lui en 1910 un transect des Alpes occidentales, du Rhône à Montgenèvre. Plus près de nous, Philippe Pinchemel consacre un film à l'agglomération parisienne et à son extension (Pathologie du paysage), en complément de nombreuses réflexions qu'il a menées sur l'aménagement du territoire et l'accroissement des villes (Pinchemel \& Pinchemel, 2005). Enfin, Fernand Verger réalise un film sur ses recherches de cartographie des littoraux assistée par la télédétection; l'action de son film se déroule dans l'Anse de l'Aiguillon, son espace laboratoire s'il en est.

Pour certains d'entre eux, ces terrains sont indissociables des maîtres qui les ont étudiés et marquent les représentations de générations de géographes. Leur postérité va jusqu'à transformer certains de ces terrains en hauts lieux, c'est-à-dire en un «lieu qui exprime symboliquement, au travers de ses représentations et de ses usages, un système de valeurs collectives ou une idéologie » (Debarbieux, 2003 : 448). Ce qui se joue sur le terrain, c'est la ritualisation d'une pratique individuelle. Si le terrain reste pour le géographe une pratique individuelle, elle devient collective lors des excursions ou lorsque la médiation du film transforme l'expérience en cas d'école, c'est-à-dire en exemple voire en emblème. C'est l'une des spécificités des hauts lieux selon Bernard Debarbieux : «Le haut lieu dispose d'un caractère symbolique dans la mesure où il naît d'un ensemble d'expériences individuelles similaires suscitées par la présence en un point et en un moment chargés de significations d'individus convaincus de partager quelque chose d'essentiel» (p. 448). Dans l'imaginaire, ces lieux restent alors associés aux maitres qui les ont étudiés et sur lesquels ils ont conduits leurs étudiants. Parfois, la ritualisation est poussée à son paroxysme: ainsi, en 1999, les participants du colloque de Rennes célébrant le centenaire de la fondation de l'Institut de géographie local ont- 
ils pu refaire la première journée de la première excursion interuniversitaire, organisée par Emmanuel de Martonne en 1905 (Baudelle, Ozouf-Marignier \& Robic (dir.), 2001). Le relief appalachien au sud de Rennes est ainsi, par la seule force de la mémoire, un haut lieu de la géographie française (et pas seulement rennaise), un lieu où souffle l'esprit du maître.

\section{Le terrain comme lieu qui commande l'accès à la communauté géographique}

La figure et le prestige du maître reposent en grande partie - conformément à l'étymologie - sur son charisme pédagogique. Le maitre est avant tout un bon enseignant qui transmet sa flamme à son auditoire ; cette formation passe généralement par la pratique répétée du terrain avec les étudiants. Le terrain est donc une instance privilégiée de formation à la géographie, un lieu où l'habitus se structure et s'acquiert. La littérature pédagogique à l'usage des étudiants, les fameux guides à l'usage des étudiants (Cholley, 1951 ; Meynier, 1971), répètent à l'envi que c'est sur le terrain que l'on apprend à faire de la géographie : « La prise de contact direct avec le paysage constitue un des meilleurs exercices pour l'aspirant géographe. Chaque fois qu'un de vos professeurs organise un tel voyage, faites l'impossible pour y assister. Cette participation doit primer les autres, réceptions, spectacles, sports. Une journée d'excursion procure plus de connaissance que dix à vingt heures de cours » (Meynier, 1971: 120). La formation au terrain ne se distingue nullement de la formation à la géographie : à l'époque, le terrain ne fait encore l'objet d'aucune réflexion théorique et méthodologique. Dans ces ouvrages - qui insistent pourtant sur sa nécessité - aucune explication n'est donnée sur la conduite du terrain. L'un et l'autre renvoient à l'exemple du maître, un modèle indispensable pour acquérir les gestes du métier.

Les modalités spécifiques de l'acquisition des méthodes du terrain, qui consistent en la seule reproduction des gestes du maître qu'il faut regarder faire et imiter en tout point, rapprochent la géographie du compagnonnage et de l'artisanat. En effet, pour le compagnon dans l'atelier comme pour l'étudiant sur le terrain, l'apprentissage consiste à reproduire l'exemple d'un maître reconnu depuis qu'il a réalisé son chef-d'œuvre qui couronne la fin de sa formation. Dans cette perspective, l'ancienne thèse d'Etat fonctionne sur le même principe que le chef d'œuvre qui couronne l'entrée à l'Université en même temps que la parfaite connaissance d'un terrain. Les analogies entre la géographie et l'artisanat ne s'arrêtent pas là : le tour de France qu'accomplit rituellement le compagnon est similaire aux excursions qui ponctuent et enrichissent la formation des étudiants.

La transmission des méthodes se fait donc sur le terrain et exclusivement par l'exemple d'un enseignant devenu pour l'occasion maître de cérémonie d'un rite initiatique qui vise à faire entrer les étudiants dans la communauté des géographes. Parfois, cette initiation prend des allures mystiques, comme le suggère Julien Gracq dans Lettrines 2 (1974) lorsqu'il fait le récit d'une excursion conduite par Emmanuel de Martonne au cours de laquelle ce dernier présente à ses étudiants qui constituent «le petit troupeau de ses vrais fidèles » un échantillon de marne verte. C'est à cette occasion que Louis Poirier, alors jeune géographe, apprend, contrairement à ses a priori, que ces marnes caractéristiques de la stratigraphie de l'Ile-de-France sont bel et bien vertes. « De Martonne s'arrêta au bord de la route pour un courte explication, puis, au flanc du fossé d'où suintait un filet d'eau, il donna deux ou trois coups de son marteau de géologue, et ramena au jour un beau morceau de glace à la pistache. J'écarquillai les yeux, comme saint Thomas devant les stigmates, et, de ce jour-là, fermement et pour toujours, je crus » (Gracq, $1995: 342)$.

Dans cette perspective, les excursions interuniversitaires apparaissent, en dépit de leur difficulté réelle, comme des moments privilégiés de sociabilité. Des étudiants avancés venus de toute la France se rencontrent une semaine durant pour découvrir une région qui leur est inconnue; ils peuvent alors bénéficier de l'enseignement des plus grands géographes du moment, qui eux aussi se retrouvent à ces occasions, même s'ils entretiennent des contacts le reste du temps 
(Wolff, 2001). Ces rencontres intergénérationnelles, qui participent de la transmission et de l'acquisition de l'habitus, se font dans une bonne humeur qui est régulièrement rappelée dans les comptes rendus. Des moments de détente sont ménagés et des chansons sont régulièrement entonnées par la caravane: "Pinchemel avait trois boutonnières, deux en France et une en Angleterre » chantaient par exemple les participants de l'excursion interuniversitaire de 1956 dans les plaines de craie du Nord de la France et du Sud-Est de l'Angleterre (Pinchemel \& Pinchemel, 2005 : ii).

Le terrain fonctionne donc comme un habitus dont la spécificité réside dans la double acception du terme terrain pour les géographes. Ainsi, le terrain désigne-t-il un ensemble de pratiques structurées et structurantes, qui conditionne l'appartenance du géographe à la communauté ; mais surtout, cet ensemble de pratiques se structure et prend sens sur le terrain, c'est-à-dire sur l'espace étudié. Cet habitus se territorialise et s'ancre dans des pratiques spatiales. C'est donc sur le terrain que l'on peut comprendre les règles de fonctionnement, de structuration de la communauté et interroger l'intégration des nouveaux membres. Ce fonctionnement spécifique de la communauté des géographes, structuré autour des pratiques du terrain et de leur transmission par les maîtres sur le terrain, garantit une certaine stabilité des pratiques ainsi que leur continuité. L'exercice du terrain semble ainsi immuable. Pourtant, pour que la géographie conserve sa pertinence, elle doit évoluer pour rendre compte de l'évolution du monde et des sociétés. Dans cette perspective, comment le terrain, pratique scientifique si structurée, peut-il évoluer et s'adapter aux évolutions du monde et par conséquent de la discipline?

\section{Le terrain : laboratoire des dynamiques disciplinaires}

Comment cet ensemble de pratiques si fortement structurées fonctionnant comme un élément fort d'identification de la communauté peut-il recevoir et intégrer les innovations disciplinaire et méthodologique? Comment cet attirail de gestes acquis et transmis peut-il s'enrichir et se renouveler pour suivre les évolutions de la discipline, sans pour autant perdre de son efficacité pratique et de sa substance identitaire ? Ces interrogations posent plus largement la question de l'évolution dans les temps d'un habitus dont le fonctionnement est décrit à un instant $t$. Comment articuler un élément statique, l'habitus, avec une vision dynamique de l'évolution des méthodes et des objets de la géographie?

\section{Le terrain : un référentiel pour comprendre les évolutions du monde}

Tout au long du XXe siècle, le monde et la société ont évolué. Leurs transformations favorisent l'apparition d'objets de recherche inédits et suscitent l'intérêt des géographes pour de nouveaux terrains. Les films pédagogiques se font l'écho de ces nouvelles préoccupations sociales et scientifiques. Ainsi, dans les années 1970, deux films posent la question de la périurbanisation et du devenir des espaces ruraux confrontés à l'étalement urbain : Pathologie du paysage (de Philippe Pinchemel et Jean-Louis Tissier) et Un tracteur de banlieue (de Bernard Kayser et Jean-Louis Tissier) sont réalisés alors que l'on observe une croissance démographique des communes rurales périphériques des agglomérations, ce qui interroge autant l'aménagement du territoire que les nouveaux modes de vie des Français ou le fonctionnement de ces espaces au paysage si caractéristique. De même, de nombreux films évoquent les transformations du monde rural (Au soleil et à l'ombre de la PAC de Jacqueline Bonnamour et Jean-Louis Tissier, Chez Claude Delhomme de Jean-Louis Tissier ou L'Espace d'un berger de Jean-Pierre Deffontaines...) ; ce fort investissement thématique pour les campagnes s'explique autant par un héritage de la géographie classique que par une forte demande sociale pour ces questions nouvelles, à une époque où le monde rural est en profonde transformation (exode rural, mécanisation, mise en place de la $\mathrm{PAC}$ et des systèmes productivistes...) et son avenir encore largement incertain. Ces films essaient à leur manière de répondre aux interrogations de la société. 
Si les évolutions du monde entraînent le renouveau des méthodes et des outils, l'apparition de nouvelles techniques ou de nouveaux modes de raisonnement peuvent aussi entraîner une modification des pratiques de terrain des géographes: ces nouveaux outils, techniques ou intellectuels, permettent aux géographes de porter un nouveau regard sur le monde. Dans Pixels et paysages, Fernand Verger utilise les premiers résultats de la télédétection pour cartographier le littoral atlantique : son film a pour but de retracer sa démarche et la place qu'y occupent ces nouveaux outils. Le terrain n'est pas abandonné et Fernand Verger vérifie in situ les résultats acquis par la machine. Le dispositif technique complète l'observation : la démarche de terrain ne change pas mais s'enrichit de nouveaux instruments de mesure. Parfois, le regard peut s'enrichir de nouveaux cadres de pensée. Ainsi, la systémique et le structuralisme font-ils leur apparition dans les raisonnements géographiques et éclairent d'un jour nouveau certains phénomènes anciennement étudiés par la discipline. Dans Physiologie du paysage, Philippe Pinchemel et Jean-Louis Tissier appliquent à un terrain connu - l'agglomération parisienne - les acquis de la systémique, ce qui permet d'envisager sous un angle nouveau les déplacements ou les flux. Les techniques évoluent et les géographes vont vers une nouvelle vision du monde et de leurs terrains.

\section{Nouveaux paradigmes et nouveau statut du terrain}

La crise de la géographie dans les années 1960 et 1970 entraîne une refondation en profondeur de la discipline et l'affirmation d'un nouveau paradigme. Les évolutions et la rupture sont exemplifiées dans ces corpus qui présentent des géographes aux postures de terrain très différenciées. Quel est l'impact de ces nouveaux paradigmes sur le terrain et sa pratique ? Loin de les remettre en cause, la refondation de la discipline et de ses méthodes a surtout pour conséquence de modifier la place de l'enquête empirique dans le processus heuristique général.

La série Analyse du paysage s'inscrit dans le cadre des recherches de Philippe Pinchemel pour recentrer la géographie autour du paysage; à ses yeux, cela implique d'énoncer une méthodologie plus rigoureuse de la description et de l'analyse paysagère qui passe notamment par l'utilisation d'un vocabulaire commun, qui permettrait d'enrichir la recherche par des comparaisons ainsi facilitées. Le premier volet de la série, Anatomie du paysage, s'inscrit dans cette démarche : loin de remettre en cause la pratique de l'autopsie, Philippe Pinchemel prône une plus grande rigueur méthodologique dans son exercice.

Cette période est aussi l'époque d'une réflexion accrue sur les concepts et les méthodes de la géographie régionale. Le découpage régional hérité de Vidal de $\mathrm{La}$ Blache fondé sur l'homogénéité des paysages et le rôle de l'histoire a fait long feu. Désormais, à la suite des travaux de Michel Rochefort et de Raymond Dugrand, les régions sont définies à partir des centres urbains qui les structurent et les polarisent. Le film d'Etienne Julliard Qu'est-ce qu'une région? Un exemple : la région de Strasbourg explique (et met en scène) en 1969 l'impasse heuristique des anciennes méthodes d'analyse régionale et propose un nouvel outillage conceptuel et pratique : désormais, l'accent est mis sur le rôle structurant des villes, ce qui se traduit par la définition de nouvelles méthodes d'enquête (en l'occurrence l'entretien avec des acteurs) qui remplacent la désormais caduque autopsie paysagère.

Les paradigmes évoluent mais la pratique du terrain, si elle est affectée, n'est pas contestée. Plus que son contenu effectif, c'est surtout sa place dans la démarche heuristique et les raisonnements qui évolue. Les comptes rendus d'excursion les plus anciens ont pour fonction principale de brosser le tableau de la région qui a accueilli la caravane. Cette démarche est inductive : les étudiants sont invités à analyser une région afin d'en dégager des phénomènes généralisables. A l'inverse, les films relèvent davantage d'une méthode déductive. Les films Pixels et paysage ou Au soleil et à l'ombre de la PAC sont révélateurs d'une nouvelle approche où l'exemple concret ne vient qu'étayer ou illustrer des règles générales formulées grâce à des 
outils modernes (télédétection, analyses de données). Deux changements interviennent sur le terrain : d'une part, le terrain n'est plus un point de départ, mais une étape dans une démarche plus complexe faite d'hypothèses et d'infirmation ou de confirmation de ces hypothèses. D'autre part, les échelles du terrain changent : la région n'est plus le niveau d'analyse pertinent, et les nouvelles recherchent s'attachent à des échelles plus grandes (comme les espaces périurbains) ou plus petites (les espaces ruraux en France).

\section{Le modèle de la greffe}

La construction de nouveaux paradigmes qui se diffusent et s'imposent a des impacts sur la fonction heuristique du terrain, mais pas sur le contenu même des pratiques déployées sur le terrain qui, elles, ne semblent pas trop évoluer. C'est du moins ce que suggère le film Stage d'étude sur le terrain en géographie de Jean Tricart qui présente une démarche pédagogique innovante qui va à rebours du fonctionnement des excursions. Alors que ces dernières constituent bien souvent un cours magistral en plein air où les étudiants sont passifs, Jean Tricart leur préfère les stages dont la longue préparation est assumée en amont par les étudiants eux-mêmes qui exploitent également à l'aval ses résultats. C'est désormais aux étudiants de construire les savoirs, avec l'aide de leur enseignant. Si la démarche d'ensemble est novatrice, le contenu des pratiques déployées sur le terrain (en l'occurrence la vallée du Rhône) est quant à lui similaire à ce qui se faisait à l'époque des excursions pourtant décriées : description de paysages, commentaires de cartes sur le terrain, coupe géologique au pied d'un talus... Dans un autre registre, les nouveaux outils comme la télédétection n'affectent pas le contenu des pratiques: dans Pixels et paysage, on voit Fernand Verger arpenter l'Anse de l'Aiguillon, y faire ses relevés... Les nouveaux outils intègrent l'attirail des méthodes de terrain, sans remettre en cause les pratiques plus anciennes, déjà éprouvées. D'une certaine manière, l'habitus du terrain intègre sans difficulté ces nouveaux outils.

Il faut proposer un modèle interprétatif pour décrire ce transfert méthodologique et l'assimilation des pratiques novatrices dans un ensemble cohérent et stabilisé comme l'habitus. Nous proposons le terme de greffe, emprunté à la botanique et au jardinage, que le Petit Robert (2004) définit ainsi : « opération par laquelle on implante un greffon », c'est-à-dire la « pousse d'une plante (...) que l'on insère dans une autre plante (porte-greffe) pour que celle-ci produise les fruits de la première ». L'habitus, structuré et structurant, est comparable au porte-greffe; sa force permet paradoxalement la vitalité de la discipline en permettant à la greffe (c'est-à-dire à l'innovation méthodologique) de prendre. Les nouveaux outils, les nouvelles méthodes - le greffon - s'intègrent à cet habitus, sans lui nuire mais au contraire en l'enrichissant et en permettant à de nouveaux fruits (des résultats scientifiques inédits) de se développer. Loin d'être des obstacles, la stabilité et l'inertie de l'habitus sont une condition essentielle de l'innovation disciplinaire et scientifique. Dans la longue durée, l'évolution se fait par des greffes successives, c'est-à-dire en assimilant progressivement les innovations ; cela permet d'expliquer à la fois les innovations continues de la géographie ainsi que la grande stabilité des pratiques de terrain dans le temps long de l'histoire de la discipline.

Ces greffes successives témoignent de la capacité d'initiative dont on dispose sur le terrain. Dans le cadre bien délimité de méthodes héritées et éprouvées, les géographes parviennent à se ménager un espace de liberté dans lequel ils peuvent mettre en œuvre des "stratégies » et des «tactiques", tracer des "chemins de traverse », à la manière de ce qu'a décrit Michel de Certeau pour les «arts de faire populaires » qui « réinventent le quotidien » (Certeau, [1980], 1990). Cette capacité d'innovation permet d'articuler le discours de la méthode à sa pratique effective : la géographie se définit alors par un ensemble de gestes qu'il faut mobiliser pour arriver à une fin. La figure du géographe oscille donc constamment entre la figure du savant qui maîtrise ces gestes et celle de l'expert praticien qui à les ruses nécessaires pour les adapter à la situation empirique de recherche. C'est donc bien sur le terrain, dans ces espaces de liberté ménagés par le chercheur, que la discipline évolue et se renouvelle. Cette part d'invention n'est 
pas théorisée : elle se cache derrière l'expérience reconnue du maître qui fonctionne ici comme une boîte noire. Seul compte le résultat positif de la recherche au détriment des arrangements effectués pour adapter la méthode aux résultats escomptés.

\section{Conclusion}

Ce recours à l'habitus pour élucider les pratiques de terrain à l'œuvre chez les géographes permet d'expliquer la pérennité de ces gestes assimilés et transmis par des générations successives de géographes; il offre ainsi un cadre opératoire pour comprendre les processus d'acquisition mis en œuvre et le fort sentiment identitaire attaché à ces pratiques. Cela met en lumière une autre composante de l'habitus bourdieusien qui apparaît en filigrane dans les lignes qui précèdent : l'articulation entre l'intériorité et l'extériorité. Dans la théorie de la socialisation formulée par Bourdieu, les pratiques témoignent de l'incorporation (c'est-à-dire d'une acquisition en profondeur) de pratiques solidement ancrées : l'acquis est intériorisé de manière à induire des pratiques; à l'inverse, les pratiques témoignent de la force de l'incorporation. Cela permet donc, dans le cas du terrain des géographes, de faire le lien entre un ancrage disciplinaire fort (permis par l'incorporation de pratiques) et un ensemble de gestes cohérents et signifiants pour celui qui appartient à la communauté et sait les décoder.

Ce binôme intériorité/extériorité peut être transposé dans le fonctionnement même de la géographie et soulève la question de la circulation des savoirs dans le champ des sciences sociales. Ce qui se joue sur le terrain, c'est la capacité qu'ont les géographes à adapter et à adopter (c'est-à-dire à intérioriser dans leur attirail méthodologique) des outils issus d'autres disciplines, tout en respectant et valorisant un héritage jugé toujours fécond. Ainsi, le terrain permet-il de distinguer deux registres différents dans les évolutions épistémologiques de la géographie: à un niveau interne, la pérennité des pratiques anciennement structurées met l'accent sur le poids de l'héritage et l'inertie de l'habitus, en dépit des évolutions conceptuelles de la discipline, alors qu'à un niveau externe, le terrain montre la capacité d'ouverture des géographes aux autres disciplines et à l'innovation méthodologique.

Cela invite à nuancer l'impact de la «crise de la géographie » comme une rupture nette dans les méthodes d'enquête utilisées ; c'est au niveau de la démarche épistémique que des changements interviennent, non dans le contenu des pratiques. Etudier le fonctionnement du terrain pendant ce «grand $\mathrm{XX}^{\mathrm{e}}$ siècle de géographie française » permet ainsi de mettre à jour des processus implicites de renouvellement disciplinaire : l'apparente faible distance critique dont on accuse souvent les géographes à l'égard de leurs objets et de leurs méthodes est largement compensée par leur forte capacité à inventer de nouvelles pratiques dans un cadre qui reste, lui, bien établi.

\section{Bibliographie}

Baudelle, G., Ozouf-Marignier, M.-V. et Robic, M.-C. (dir.) (2001). Géographes en pratiques (1870-1945). Le terrain, le livre, la cité. Rennes : PUR. 390 p.

Bourdieu, P. (1980). Le sens pratique. Paris : Les éditions de Minuit. 479 p.

Calbérac, Y. (2005). «En quête du terrain. Approche historique et épistémologique du terrain en géographie ». Mémoire de Master de géographie dirigé par Mme Isabelle Lefort. Ecole normale supérieure Lettres et Sciences humaines. $122 \mathrm{p}$.

Certeau, M. de ([1980] 1990). L'invention du quotidien. 1 : Arts de faire. Paris : Gallimard. 350 p.

Cholley, A. ([1942] 1951). La géographie. Guide de l'étudiant. Deuxième édition. Paris : PUF. $218 \mathrm{p}$.

Debarbieux, B. (2003). « Haut lieu » in Lévy, J. et Lussault, M. (dir.) (2003). Dictionnaire de la géographie et de l'espace des sociétés. Paris : Belin. 1034 p. p. 448-449. 
Dubost, M. (2004). « Le Centre Audio-Visuel de Saint-Cloud et ses origines » in Pastre-Robert, B. de, Dubost, M. et Massit-Folléa, F. (dir.) (2004). Cinéma pédagogique et scientifique. A la redécouverte des archives. Lyon : ENS Editions. 136 p. p. 39-44.

Gracq, J. (1995). Euvres complètes (tome 2). Paris : Gallimard. Bibliothèque de la Pléiade. $1756 \mathrm{p}$.

Latour, B. (1993). «Portrait d'un biologiste en capitaliste sauvage» in Petites leçons de sociologie des sciences. Paris : Le Seuil. 253 p. p.100-129.

Latour, B. (2005). La science en action. Introduction à la sociologie des sciences. Paris : La Découverte. $663 \mathrm{p}$.

Lefort, I. et Pelletier, P. (2006). Grandeurs et mesures de l'écoumène. Paris : Economica. 229 p. Meynier, A. (1971). Guide de l'étudiant en géographie. Paris : PUF. 158 p.

Pinchemel, G. et Pinchemel, P. (2005). Géographes. Une intelligence de la terre. Paris : Editions Arguments. 295 p.

Robic, M.-C. (1996). "Interroger le paysage ? L'enquête de terrain, sa signification dans la géographie humaine moderne (1900-1950) », in Blanckaert, C. (dir.). Le terrain des sciences humaines (XVIII ${ }^{e}-X X^{e}$ siècle). Paris : L'Harmattan. 404 p. p. 357-388.

Robic, M.-C. (dir.) (2006). Couvrir le monde. Un grand XX siècle de géographie française. Paris : ADPF. 232 p.

Volvey, A. (2003). «Terrain» in Lévy, J. et Lussault, M. (dir.) (2003). Dictionnaire de la géographie et de l'espace des sociétés. Paris : Belin. 1034 p. p. 904-906.

Wolff, D. (2001). «A travers les correspondances : l'envers ou l'enfer de l'excursion... » in Baudelle, G., Ozouf-Marignier, M.-V. et Robic, M.-C. (dir.). Géographes en pratiques (18701945). Le terrain, le livre, la cité. Rennes : PUR, 390 p. p. 329-342.

\section{Filmographie}

Bonnamour, J. et Tissier, J.-L. (1993). Au soleil et à l'ombre de la PAC. Dynamiques spatiales de la modernisation agricole en France. Réalisateur : J.-C. Durand. 20 min.

Deffontaines, J.-P. et Landais, E. (1989). L'espace d'un berger. Réalisateur : D. Garabédian. 60 min.

Julliard, E. (1969). Qu'est-ce qu'une région? Un exemple: la région de Strasbourg. Réalisateur : P. Samson. 40 min.

Kayser, B., Labory, J.-P. et Tissier, J.-L. (1980). Un tracteur de banlieue. Réalisateur: P. Samson. $27 \mathrm{~min}$.

Pinchemel, P. et Tissier, J.-L. (1978). Analyse du paysage. 1: Anatomie du paysage. Réalisateur: P. Samson. 28 min.

Pinchemel, P. et Tissier, J.-L. (1979). Analyse du paysage. 2: Physiologie du paysage. Réalisateur : P. Samson. 22 min.

Pinchemel, P. et Tissier, J.-L. (1986). Analyse du paysage. 4: Pathologie du paysage. Réalisateur : P. Samson. 22 min.

Tissier, J.-L. (1982). Des géographes. Réalisateur : J.-L. Tissier. 31 min.

Tissier, J.-L. (1993). Du côté de Ferrière avec Claude Delhomme. Réalisateur : J.-C. Durand. $16 \mathrm{~min}$.

Tricart J. et Bastid J.-P. (1973). Stage d'étude sur le terrain en géographie. Réalisateur: G. Poinssac. $27 \mathrm{~min}$.

Verger, F. et Tissier, J.-L. (1985). Pixels et paysages. Réalisateur : J.-C. Durand. 24 min. 